ENCYCLOPEDDIE Encyclopédie berbère

BERBERE

$31 \mid 2010$

31 | Matmora - Mezrag

\title{
Mercenaires (Guerre des)
}

\section{T. Kotula}

\section{OpenEdition}

Journals

Édition électronique

URL : http://journals.openedition.org/encyclopedieberbere/561

DOI : 10.4000/encyclopedieberbere.561

ISSN : 2262-7197

\section{Éditeur}

Peeters Publishers

\section{Édition imprimée}

Date de publication : 30 décembre 2010

Pagination : 4871-4874

ISBN : 978-90-429-2368-3

ISSN : 1015-7344

Référence électronique

T. Kotula, « Mercenaires (Guerre des) », Encyclopédie berbère [En ligne], 31 | 2010, document M91, mis en ligne le 08 octobre 2020, consulté le 12 octobre 2020. URL : http://journals.openedition.org/ encyclopedieberbere/561; DOI : https://doi.org/10.4000/encyclopedieberbere.561

Ce document a été généré automatiquement le 12 octobre 2020

(c) Tous droits réservés 


\section{Mercenaires (Guerre des)}

\section{T. Kotula}

1 Il est devenu traditionnel de présenter sous le nom de guerre des mercenaires le mouvement des mercenaires et des indigènes africains qui se sont révoltés contre Carthage après la première guerre punique. C'est la participation de ces derniers qui mérite ici une attention particulière. Ce grave conflit interne si dangereux pour l'Etat

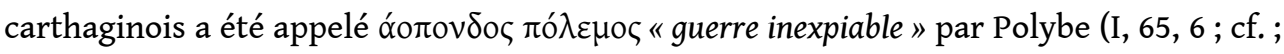

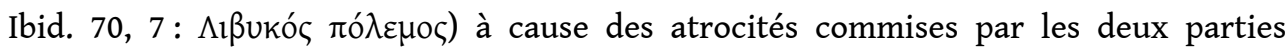
belligérantes. Polybe, notre source principale, lui a consacré un ample récit (I, 65-88) dont le fond est sans doute emprunté à un historien grec plus ancien (Philinos d'Agrigente ?) sympathisant d'Hamilcar Barca et hostile aux insurgés. Il y a peu à tirer des courts passages de Diodore (XXV, 2-6; 8) ; Cornelius Nepos (Ham. 2) ; Tite-Live (XXI, 1,$4 ; 2,1 ; 41,12$ ) ; Appien (Sic. II, 3 ; Pun. 5 ; lbar. 4) ; Zonaras (VIII, 17).

2 D’après Polybe, la révolte résultait du mécontentement des mercenaires dont la solde demeurait impayée, la guerre perdue en Sicile ayant épuisé le trésor punique. Le gouvernement carthaginois s'est débarrassé des importuns, dont la multitude constituait une grave menace pour la capitale, les faisant partir pour Sicca (El Kef). Mais leurs demandes n'ayant pas été satisfaites, ils ont quitté Sicca et, irrités contre les Carthaginois, ne se sont arrêtés que devant Tunis. C'était là, à une vingtaine de kilomètres de la capitale, que le gouvernement dut négocier avec les mercenaires. Giscon, chef punique commandant la garnison de Lylibée en Sicile et à l'égard duquel les mercenaires étaient bien disposés, fut chargé de commencer le règlement des arriérés, mais les moyens dont il disposait se révélèrent insuffisants. Une grave émeute ayant éclaté dans le camp, les rebelles ont emprisonné Giscon et sa suite et ont élu comme chefs de la révolte un certain Spendios, ancien esclave campanien, et un Libyen*, Mathos. Ce dernier représentait des mercenaires indigènes qui constituaient au sein des troupes révoltées l'élément prédominant. Mathos commença par gagner à la cause commune les paysans libyens opprimés par Carthage et même les Numides*, et bientôt le mouvement devient général. Carthage même se trouva bloquée et les villes voisines d'Utique et d'Hippo Diarrhytos (Bizerte) furent assiégées. De graves défaites furent infligées aux Puniques par les insurgés dont le nombre s'accrût 
considérablement grâce à un afflux massif d'Africains. Ce fut alors, dans le danger suprême, que les Carthaginois se décidèrent à confier le commandement unique à Hamilcar Barca. Ce général, dont le nom et la stratégie étaient devenus célèbres en Sicile, réorganisa l'armée qui, avec sa cavalerie et surtout avec ses éléphants, répandait la terreur parmi les indigènes. Grâce à un stratagème habile, il débloqua Carthage ensuite, après avoir obtenu le concours du chef de cavaliers numides Naravas (Polybe, I : 78), il battit le corps de Spendios. En pratiquant une politique de mansuétude à l'égard des vaincus et des prisonniers, il visait la défection d'une partie au moins des insurgés. A cette indulgence, les trois généraux rebelles, Mathos, Spendios et Autaritos - chef des mercenaires gaulois - répondirent par des actes d'atrocité, pour rendre toute réconciliation impossible. Giscon et des centaines de prisonniers furent mis à mort. Ce fut dès lors que les opérations menées sans pitié de part et d'autre valurent à la guerre le nom d'óo

3 Malgré la reddition d'Utique et d'Hippo aux rebelles, la fortune continuait à favoriser Hamilcar. Il réussit à cerner et à assiéger les ennemis qui, en proie à une grande famine, durent se livrer au cannibalisme. S'étant emparé par ruse des dix députés mercenaires, dont Spendios, qui s'étaient présentés à lui pour traiter de la paix, le général punique put écraser le gros de l'armée rebelle (plus de 40000 hommes selon Polybe, I, 85, 7) dans le défilé de la Scie.

4 Pendant ce temps, le libyen Mathos avait plus de chance. Bien que bloqué entre Tunis et Carthage, il surprit et anéantit par une attaque hardie un corps punique, ce qui lui permit de s'évader dans l'intérieur du pays. Mais finalement, dans une bataille décisive près de Leptis Minor, le reste de son armée fut vaincu et lui-même capturé. Bientôt Utique et Hippo Diarrhytos durent se rendre à la merci des Puniques et la guerre des mercenaires prit fin, après trois ans et quatre mois.

5 Tel est, en bref résumé, l'essentiel des faits relatés par Polybe. Mais malgré son ampleur considérable, ce récit bien connu, qui a pour but de mettre en relief de grands exploits d'Hamilcar, laisse beaucoup de points obscurs.

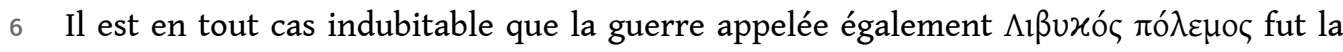
révolte la plus grave des Africains contre le pouvoir de Carthage. Polybe est suivi par d'autres auteurs dans son opinion que la guerre « inexpiable » était un phénomène très complexe. Quant à la participation des indigènes qui se sont joints aux mercenaires, il est évident que cette composante africaine du mouvement contribue à prouver que les puniques ont exploité leurs sujets libyens, paysans autochtones libres, d'une façon très dure. On peut l'inférer de Polybe I, 71-72 (surtout 72.2). On apprend que les Libyens étaient exaspérés puisqu'ils durent donner, au temps de la première Guerre punique, la moitié de leur récolte en tribut. Simultanément, l'Etat exigea des citadins des tributs doubles de ceux qu'ils payaient auparavant. On peut donc en conclure que le taux normal des redevances imposées aux cultivateurs libyens était du quart de leur revenu en blé. Le doublement des taxes a rendu leur situation critique et la révolte, inévitable.

7 Mais il est à signaler que, en plus des Libyens, certains Numides coopérèrent à la révolte africaine de façon très active d'après nos sources (Polybe, I, 65, 77, 78 ; Appien, Sic. II, 3). Il s'agit, selon toute vraisemblance, des tribus massyles voisines de l'Etat carthaginois et menacées par l'expansion punique. C'est sans doute le danger punique qui contribua, dans une certaine mesure, à la formation du royaume massyle des ancêtres de Massinissa*. Vers 220 avant notre ère, Gaïa*, père de Massinissa, a même occupé un territoire carthaginois qu'il conserva assez longtemps. Au cours de la 
" guerre inexpiable » Naravas, chef d'un clan ou d'une tribu numide, passa du camp des mercenaires à celui d'Hamilcar avec un corps de cavalerie. Cette trahison a montré, encore une fois, que Carthage savait toujours gagner des indigènes à sa cause. Mais d'autres Numides ont dû être combattus, même après la défaite de Mathos. Ce fut une campagne punitive en Numidie qui termina la révolte (Diodore, XXVI, 23).

En l'Etat actuel des connaissances, la chronologie des évènements demeure incertaine. Polybe n'indique que la durée totale de la campagne : trois ans et quatre mois (I, 88, 7 ; mais Diodore, XXV, 6 : quatre ans et quatre mois ; Tite-Live, XXI, 2, 1 propose un chiffre arrondi de cinq ans). Les historiens modernes admettent le plus souvent les limites automne 241 (depuis la paix en Sicile), - 238; ou fin 241 - début 237 (dernière campagne en Numidie). A plus forte raison restent hypothétiques toute les supputations concernant la chronologie interne de la révolte.

De graves incertitudes surgissent également au sujet des derniers faits narrés par Polybe. Sa source principale semble l'avoir déçu sur ce point, puisque son récit, prolixe jusque là, devient ensuite très laconique et superficiel. Cela vaut aussi bien pour la description de la bataille finale que pour sa topographie (région de Leptis, assurément Minor, I, 87). D'ailleurs, Polybe est également peu précis dans l'indication du lieu de la destruction des troupes de Spendios par Hamilcar : «lien que l'on appelle la Scie » (I, 85, 7). Cette information ne paraît pas permettre d'identifier la Scie $(\pi \rho \omega v)$ d'une manière certaine.

De même, on peut objecter à Polybe, ou plutôt à sa source, une forte exagération dans l'évaluation des effectifs de l'armée insurgée. Il résulte de deux passages que l'on trouve chez cet historien, que Mathos et Spendios ont rassemblé jusqu'à cent mille hommes (plus de vingt mille mercenaires et soixante-dix mille Africains; Polybe I, 67, 73 ; cf. Cornelius Nepos Ham. 2, 2: amplius centum milia armatorum). D'après le même auteur, les forces carthaginoises composées de tous les effectifs disponibles (les mercenaires nouvellement enrôlés compris) et confiées à un chef unique n'ont atteint qu'un total de dix mille fantassins et cavaliers (ibid. 75, cf. 76). La disproportion saute aux yeux et il est évident que le récit a été composé ad maiorem gloriam du vainqueur Hamilcar Barca.

11 Mais il faut constater en même temps que les spéculations modernes ayant pour but de corriger des chiffres transmis par les auteurs anciens n'ont pas abouti à des résultats satisfaisants. Par contre, les savants modernes ont à juste titre insisté sur les aspects sociaux de la "guerre inexpiable ». C'était surtout la participation massive des paysans libyens opprimés, leur lutte contre l'aristocratie terrienne punique, qui donna à la mutinerie des mercenaires le caractère d'un mouvement social. Mais il est peu probable que les esclaves aient pu jouer un rôle bien considérable dans les opérations militaires en Afrique. Polybe n'en dit rien et les informations tardives d'Appien (Sic. II, 3 : grand nombre d'esclaves fugitifs) et à plus forte raison de Zonaras (VIII, 17) sont très vagues et fort suspectes.

12 Compte tenu que la majorité des mercenaires étaient les Libyens commandés par leur propre chef indigène - Mathos - et que celui-ci a su inspirer et organiser une révolte générale des Africains contre Carthage, la "guerre inexpiable» doit être appréciée avant tout comme un de ces soulèvements autochtones si typiques de l'histoire nordafricaine. 


\section{BIBLIOGRAPHIE}

CAMPS G., 1960 - Aux origines de la Berbérie. Massinissa ou les débuts de l'Histoire, Libyca Archéol.

Epigr.t. 8.

CHARLES - PICARD G., 1967 - Hannibal, p. 67-74, Paris.

CHARLES - PICARD G. et C., 1958 - La vie quotidienne à Carthage au temps d'Hannibal, Paris, p. 123-125.

COMBET-FARNOUX B., 1960 - Les guerres puniques, Coll. Que sais-je ? nº 888, Paris, p. 52-55.

EHRENBERG V., 1930 - « Mathos », in Paulys-Wissowa, Realencyclopädie, XIV, 2, col. 2195-2197.

GSELL ST., 1921 - Histoire ancienne de l'Afrique du Nord, t. III, Paris. p. 100-126.

котULA T., Malo znane powstanie Afrow i niewolnikow przeciw Kartaginie, (Diod. Sic., XIV, 77), Une révolte peu connue des Africains et des esclaves contre Carthage, Meander, t. 21, p. 362-371. KROMAYER J. et VEITH G., 1912 - Antike Schlachtfelder, III, 2, Berlin, p. 519-571. MACHKINE N. A., Le dernier siècle de la Carthage punique, Vestnik Drevnej Istorii, fasc. 2, p. 43-44, Paris.

MELTZER O., 1896 - Geschichte der Karthager, t. II, Berlin, p. 40-41, 360-387, 589-590.

MUNZER F., 1930 - « Spendius », in Paulys-Wissowa, Realencyclopädie, III, A 2, col. 1610.

SANCTIS G. de., 1930 - Storia dei Romani, t. III, 1, Firenze, p. 130-149.

WALTER G., 1947 - La destruction de Carthage 264-146 av. J-C., Paris, p. 228-254.

WARMINGTON B. H., 1961 - Histoire et civilisation de Carthage (814 av. J. C. à 146 av. J. C.), Paris, p. $240-244$.

INDEX

Mots-clés : Antiquité, Carthage, Histoire militaire 